\title{
Can electron distribution functions be derived through the Sunyaev-Zel'dovich effect?
}

\author{
D. A. Prokhorov ${ }^{1,2,3}$, S. Colafrancesco ${ }^{4,5}$, T. Akahori ${ }^{6}$, K. Yoshikawa ${ }^{7}$, S. Nagataki ${ }^{3}$, and K.-I. Seon ${ }^{1}$ \\ 1 Korea Astronomy and Space Science Institute, Hwaam-dong, Yuseong-gu, Daejeon 305-348, Republic of Korea \\ e-mail: phdmitry@stanford.edu \\ 2 Hansen Experimental Physics Laboratory, Kavli Institute for Particle Astrophysics and Cosmology, Stanford University, Stanford, \\ CA 94305, USA \\ 3 YITP, Kyoto University, Kyoto 606-8502, Japan \\ 4 INAF - Osservatorio Astronomico di Roma via Frascati 33, 00040 Monteporzio, Italy \\ e-mail: sergio.colafrancesco@oa-roma.inaf.it \\ 5 ASI V.le Liegi 26, Roma, Italy \\ e-mail: Sergio.Colafrancesco@asi.it \\ ${ }^{6}$ Research Institute of Basic Science, Chungnam National University, Daejeon, Republic of Korea \\ 7 Center for Computational Sciences, University of Tsukuba, 1-1-1, Tennodai, Ibaraki 305-8577, Japan
}

Received 31 October 2010 / Accepted 8 February 2011

\begin{abstract}
Aims. Measurements of the Sunyaev-Zel'dovich (hereafter SZ) effect distortion of the cosmic microwave background provide methods to derive the gas pressure and temperature of galaxy clusters. Here we study the ability of SZ effect observations to derive the electron distribution function (DF) in massive galaxy clusters.

Methods. Our calculations of the SZ effect include relativistic corrections considered within the framework of the Wright formalism and use a decomposition technique of electron DFs into Fourier series. Using multi-frequency measurements of the SZ effect, we find the solution of a linear system of equations that is used to derive the Fourier coefficients; we further analyze different frequency samples to decrease uncertainties in Fourier coefficient estimations.

Results. We propose a method to derive DFs of electrons using SZ multi-frequency observations of massive galaxy clusters. We found that the best frequency sample to derive an electron DF includes high frequencies $v=375,600,700,857 \mathrm{GHz}$. We show that it is possible to distinguish a Juttner DF from a Maxwell-Bolzman DF as well as from a Juttner DF with the second electron population by means of SZ observations for the best frequency sample if the precision of SZ intensity measurements is less than $0.1 \%$. We demonstrate by means of 3D hydrodynamic numerical simulations of a hot merging galaxy cluster that the morphologies of SZ intensity maps are different for frequencies $v=375,600,700,857 \mathrm{GHz}$. We stress that measurements of SZ intensities at these frequencies are a promising tool for studying electron distribution functions in galaxy clusters.
\end{abstract}

Key words. galaxies: clusters: intracluster medium - relativistic processes - cosmic background radiation

\section{Introduction}

Massive clusters of galaxies are the largest virialized objects in the Universe bound by gravitation in the presence of dark matter. Apart from galaxies and dark matter, galaxy clusters contain a hot highly ionized plasma with temperatures up to $15 \mathrm{keV}$ that emits in X-rays: a continuum through bremsstrahlung and lines through spontaneous decays of excitation states of ions. The hot intracluster plasma can be fully described by the particle distribution function (DF).

Equilibrium DFs are different in non-relativistic and relativistic statistics. A thermal equilibrium DF is described by Maxwell-Boltzman and Juttner functions in the framework of the non-relativistic and relativistic theories, respectively (Landau \& Lifshitz 1976). High-energy phenomena allow us to study thermal mildly relativistic particle populations, such as mildly relativistic electron populations in clusters of galaxies.

Inverse Compton (IC) scattering of hot free electrons in clusters of galaxies on the cosmic microwave background (CMB) radiation field is another effect that provides us with a method to study hot plasmas in galaxy clusters, because IC scattering causes a change in the intensity of the $\mathrm{CMB}$ radiation toward clusters of galaxies (the Sunyaev-Zel'dovich effect, hereafter the SZ effect; for a review, see Sunyaev \& Zel'dovich 1980), which depends on the details of the electron distribution function in the cluster atmosphere (see, e.g., Colafrancesco et al. 2003).

A relativistically correct formalism for the $\mathrm{SZ}$ effect based on the probability distribution of the photon frequency shift after scattering was given by Wright (1979) to describe the Comptonization process of soft photons by mildly relativistic plasma. Relativistic corrections for the SZ effect allow us to measure the temperature of intracluster plasma (see, e.g., Pointecouteau et al. 1998; Hansen et al. 2002) and have been studied both analytically (Colafrancesco et al. 2003; Colafrancesco \& Marchegiani 2010) and by means of numerical simulations (Prokhorov et al. 2010a).

There are different methods proposed so far to derive a plasma temperature by means of the SZ effect, which are based on the measurement of one of following quantities: the shift of the crossover frequency (see Rephaeli 1995), the intensity slope 
around the crossover frequency (see Colafrancesco et al. 2009), the wide frequency spectroscopy of the SZ effect spectrum especially at high frequencies (Colafrancesco \& Marchegiani 2010), and the ratio of the SZ intensities at two frequencies (Prokhorov et al. 2010a). As was noticed by Rephaeli (1995) the correct relativistic equilibrium distribution is essential for the proper interpretation of measurements of the SZ effect.

One of the methods to study the relativistic equilibrium DF of particles is to perform fully relativistic molecular dynamics simulations (see Cubero et al. 2007, for the 1D case; Montakhab et al. 2009, for the 2D case; and Peano et al. 2009, for the 3D case). However, to justify the use of the relativistic equilibrium DF in astrophysics, an observational confirmation is required.

In this paper we propose a method to derive the relativistic equilibrium DF that is based on multi-frequency measurements of the SZ effect in massive clusters. This provides us with a more complete analysis of the electron distribution in the velocity space than that given by knowing only the temperature value, and a method to verify the relativistic equilibrium DF. We also check the validity of using the Juttner DF as an appropriate approximation to the universal (equilibrium) electron distribution in massive merging clusters.

Hard X-ray emission tails reported in Beppo-SAX and RXTE X-ray spectra of some galaxy clusters (see FuscoFemiano et al. 1999, 2004; Rossetti \& Molendi 2004, for the Coma cluster; Petrosian et al. 2006, for the Bullet cluster) were interpreted as bremsstrahlung emission from non-thermal subrelativistic electrons (e.g. Sarazin \& Kempner 2000) or from thermal electrons with a Maxwellian spectrum distorted by a particle acceleration mechanism (Blasi 2000; Liang et al. 2002), or from thermal electrons with a Maxwellian spectrum with a high temperature (e.g., Petrosian et al. 2006). Million \& Allen (2009) reported the discovery of spatially extended, non-thermal-like emission components in Chandra X-ray spectra for five massive galaxy clusters. Using Swift/BAT data, Ajello et al. (2010) have confirmed the presence of a hard X-ray excess from the Bullet cluster. Note that a possible contribution from inverse Compton emission of highly relativistic electrons with Lorentz factor $\gamma \sim 10^{4}$ to a hard X-ray excess (for a review, see Rephaeli et al. 2008) constrains our possibilities to study electrons DFs by using X-ray observations. It has been suggested that a good test to check the bremsstrahlung interpretation of hard X-ray tails is to use multi-frequency measurements of the SZ effect (see Dogiel et al. 2007).

Spatially-resolved observations of the SZ effect can also provide relevant information on the distribution of the electron plasma in galaxy clusters with various temperatures. Prokhorov et al. (2010a) found that the morphologies of the SZ intensity maps of a cool galaxy cluster at frequencies of $128 \mathrm{GHz}$ and $369 \mathrm{GHz}$ are similar. Here we perform numerical simulations of a hot merging galaxy cluster to study the morphologies of the SZ intensity maps for a hot merging galaxy cluster to explore the different morphologies for low and high frequencies that depend on the importance of the SZ effect relativistic corrections.

The layout of the paper is as follows. The dependence of the $\mathrm{CMB}$ distortion caused by the SZ effect on the electron DF is considered in Sect. 2 in the framework of the relativistic correct Wright formalism. We propose a method to derive a velocity DF from multi-frequency SZ observations in Sect. 3. Relaxation of a system of electrons to equilibrium distributions is considered in Sect. 4. We estimate the precision of SZ observations, which is necessary to derive the electron DF proposed as an explanation of hard tails in X-ray spectra of galaxy clusters, in Sect. 5. Using 3D hydrodynamic numerical simulations of a hot merging galaxy cluster and the Wright formalism, we demonstrate that the morphologies of the SZ intensity maps are different at low and high frequencies in Sect. 6. We present the observational estimates and discuss the confusion noises in Sect. 7. We discuss our results and present our conclusions in Sect. 8.

\section{Dependence of the $\mathrm{CMB}$ distortion caused by the SZ effect on the DF of electrons}

In this section we discuss the difference between the CMB distortions (caused by the SZ effect) that are caused by a departure from the diffusive approximation given by Kompaneets (1957) from those that are caused by using a relativistic correct DF instead of a Maxwell-Boltzman DF.

In the diffusion approximation and for a non-relativistic electron population, the $\mathrm{CMB}$ intensity change caused by the SZ effect is

$\Delta I_{\mathrm{nr}}=I_{0} \tau \frac{k_{\mathrm{B}} T_{\mathrm{e}}}{m_{\mathrm{e}} c^{2}} g_{\mathrm{nr}}(x)$

where $I_{0}=2\left(k_{\mathrm{B}} T_{\mathrm{CMB}}\right)^{3} /(h c)^{2}, T_{\mathrm{e}}$ is the electron temperature, $\tau$ is the the scattering optical depth, $m_{\mathrm{e}}$ the electron mass, $c$ the speed of light, $k_{\mathrm{B}}$ the Boltzmann constant, $x=h v /\left(k_{\mathrm{B}} T_{\mathrm{CMB}}\right), h$ the Planck constant, and the spectral function $g_{\mathrm{nr}}(x)$ is given by

$g_{\mathrm{nr}}(x)=\frac{x^{4} \mathrm{e}^{x}}{\left(\mathrm{e}^{x}-1\right)^{2}}\left(x \cdot \frac{\mathrm{e}^{x}+1}{\mathrm{e}^{x}-1}-4\right)$.

The subscript "nr" denotes that the previous expression was obtained in the non-relativistic limit.

In the relativistic treatment taking into account the scattering to arbitrary frequencies (Wright 1979), the CMB spectral distortion caused by the SZ effect is a functional of the electron velocity $\mathrm{DF}$ and is given by

$\Delta I\left[x, f_{\mathrm{e}}\right]=I_{0} \tau \int \mathrm{d} s P_{1}\left[s, f_{\mathrm{e}}\right]\left(\frac{x^{3} \mathrm{e}^{-3 s}}{\exp \left(x \mathrm{e}^{-s}\right)-1}-\frac{x^{3}}{\mathrm{e}^{x}-1}\right)$

with

$P_{1}\left[s, f_{\mathrm{e}}\right]=\int \mathrm{d} \beta f_{\mathrm{e}}(\beta) P(s, \beta)$

where $P_{1}\left[s, f_{\mathrm{e}}\right]$ is the probability distribution of a scattering frequency shift that is a functional of the electron velocity $\mathrm{DF}, f_{\mathrm{e}}(\beta)$, of the electron population and $P(s, \beta) \mathrm{d} s$ is the probability that a single scattering of a CMB photon off an electron with speed $\beta c$ causes a logarithmic frequency shift $s \equiv \ln \left(v^{\prime} / v\right)$, and $\beta=V / c$, where $V$ is the electron velocity.

We notice that this formalism is valid in the single scattering approximation and for low values of the optical depth $\tau$, which is however sufficient for the purposes of this paper. A more general description of the CMB spectral distortions caused by the SZ effect can be found in Colafrancesco et al. (2003).

The SZ intensity spectra $G(x)=\Delta I(x) /\left(I_{0} y\right)$, where $y$ is the Comptonization parameter, derived in the framework of the Wright formalism assuming Juttner and Maxwell-Boltzman DFs are shown in Fig. 1 for a massive cluster with a temperature of $15.3 \mathrm{keV}$. The SZ intensity spectrum in the Kompaneets approximation is also shown in Fig. 1.

To study the effects of the CMB intensity change caused by the SZ effect that is in turn caused by a departure from the diffusive approximation and by using a relativistic correct DF instead of a Maxwell-Boltzman DF, we calculate the ratios 
D. A. Prokhorov et al.: Can electron distribution functions be derived via the SZ effect?

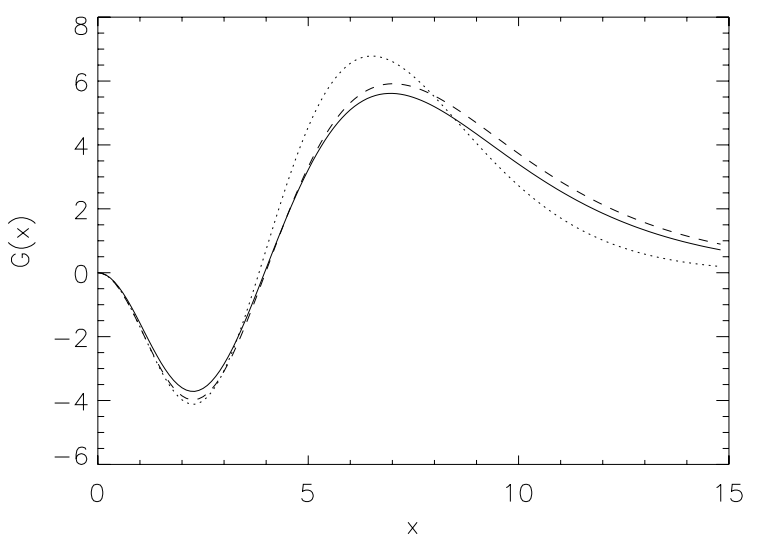

Fig. 1. SZ intensity spectra $G(x)=\Delta I(x) /\left(I_{0} y\right)$ for a massive cluster with a temperature of $15.3 \mathrm{keV}$ for Juttner and Maxwell-Boltzman DFs shown by the solid and dashed lines, respectively. The SZ intensity spectrum in the Kompaneets approximation is shown by the dotted line.

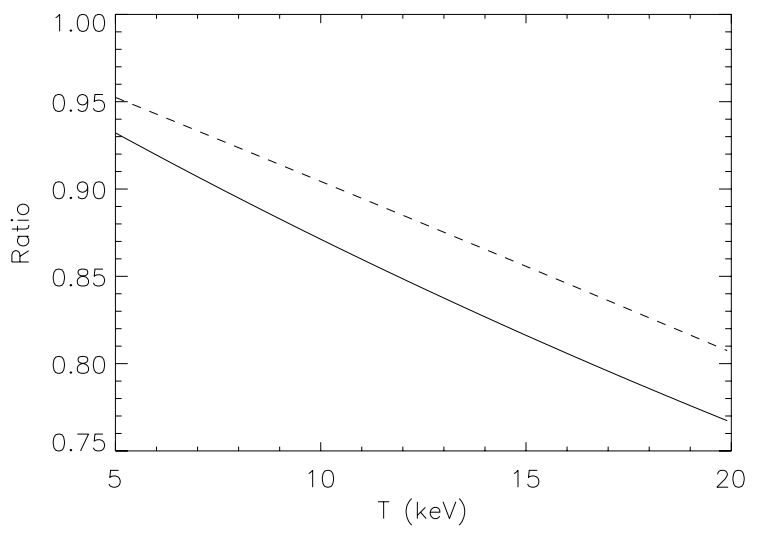

Fig. 2. Ratios $\Delta I\left[f_{\mathrm{J}}(\beta)\right] / \Delta I_{\mathrm{nr}}$ and $\Delta I\left[f_{\mathrm{M}}(\beta)\right] / \Delta I_{\mathrm{nr}}$ as functions of the electron temperature are shown by the solid and dashed lines, respectively.

$\Delta I\left[f_{\mathrm{J}}(\beta)\right] / \Delta I_{\mathrm{nr}}$ and $\Delta I\left[f_{\mathrm{M}}(\beta)\right] / \Delta I_{\mathrm{nr}}$ as functions of the electron temperature, where $f_{\mathrm{J}}(\beta)$ and $f_{\mathrm{M}}(\beta)$ are the Juttner and MaxwellBoltzman DFs, respectively. These ratios are shown in Fig. 2 for Juttner and Maxwell-Boltzman DFs by solid and dashed lines, respectively, at a frequency of $369 \mathrm{GHz}$, the choice of this frequency is reasonable since the relativistic corrections of the SZ effect are larger at high frequencies.

To qualify the CMB intensity change caused by the SZ effect that is in turn caused by using a relativistic correct DF instead of a Maxwell-Boltzman DF, we calculate the ratio of the CMB intensity change caused by the SZ effect that is in turn caused by using a relativistic correct DF instead of a Maxwell-Boltzman DF to that given by the total contribution of the SZ relativistic corrections. This ratio is given by the expression

$D=\frac{\Delta I\left[f_{\mathrm{M}}(\beta)\right]-\Delta I\left[f_{\mathrm{J}}(\beta)\right]}{\Delta I_{\mathrm{nr}}-\Delta I\left[f_{\mathrm{J}}(\beta)\right]}$

and is shown (in\%) at a frequency of $369 \mathrm{GHz}$ in Fig. 3 as a function of temperature. Figure 3 shows that the correction from using a relativistic correct DF instead of a Maxwell-Boltzman $\mathrm{DF}$ is a more significant fraction of the total contribution of the SZ relativistic corrections at lower temperatures. However, we stress that the value of the CMB intensity change because of using a relativistic correct DF instead of a Maxwell-Boltzman DF will be much higher in hot galaxy clusters and, therefore, measurements of the relativistic SZ corrections in hot clusters

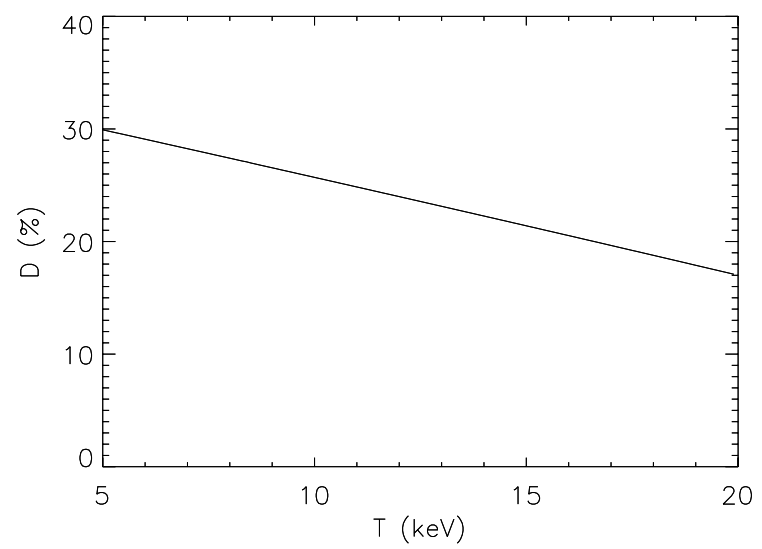

Fig. 3. Ratio of the CMB intensity change caused by the SZ effect caused by using a relativistic correct DF instead of a MaxwellBoltzman DF to that given by the total contribution of the SZ relativistic corrections.

will be more effective to probe the electron DF. This is because the value of the relativistic $\mathrm{SZ}$ corrections is proportional to $T_{\mathrm{e}}^{5 / 2}$, since $\tau \propto \sqrt{T_{\mathrm{e}}}$ (e.g., Bryan \& Norman 1998) and

$$
\int \mathrm{d} s P_{1}\left[s, f_{\mathrm{e}}\right]\left(\frac{x^{3} \mathrm{e}^{-3 s}}{\exp \left(x \mathrm{e}^{-s}\right)-1}-\frac{x^{3}}{\mathrm{e}^{x}-1}\right)-\frac{k_{\mathrm{B}} T_{\mathrm{e}}}{m_{\mathrm{e}} c^{2}} g_{\mathrm{nr}}(x) \propto T_{\mathrm{e}}^{2},
$$

see, e.g., Challinor \& Lasenby (1998). Therefore, the relativistic SZ corrections for a galaxy cluster with a gas temperature of $15 \mathrm{keV}$ will be in $\approx 16$ times larger than that for a galaxy cluster with a gas temperature of $5 \mathrm{keV}$. Because the second-order and third-order (in the expansion parameter $\Theta=k_{\mathrm{B}} T_{\mathrm{e}} /\left(m_{\mathrm{e}} c\right)$ ) relativistic effects make a significant contribution to the SZ spectral distortion for $k_{\mathrm{B}} T_{\mathrm{e}} \approx 15 \mathrm{keV}$ (Challinor \& Lasenby 1998; Itoh et al. 1998), we checked the relativistic SZ corrections for a hot galaxy cluster by using the Wright formalism and found that the relativistic SZ corrections at frequencies of $369 \mathrm{GHz}$ and $857 \mathrm{GHz}$ for a galaxy cluster with a gas temperature of $15 \mathrm{keV}$ are $\approx 14$ and $\approx 17$ times larger than those at frequencies of $369 \mathrm{GHz}$ and $857 \mathrm{GHz}$ for a galaxy cluster with a gas temperature of $5 \mathrm{keV}$, respectively. This agrees with the result obtained above.

Because the SZ intensity change from using a relativistic correct DF instead of a Maxwell-Boltzman DF is a significant fraction of the total contribution of the SZ relativistic corrections (see Fig. 3), we conclude that this should provide us with the ability to derive an electron DF by means of the SZ effect. In the following section we propose a method to derive a DF of electrons from multi-frequency SZ observations.

\section{A method to derive a velocity DF from multi-frequency SZ observations}

We use a Fourier analysis to derive a DF of electrons from multifrequency SZ observations and to find the best frequencies at which this method can be successfully applied.

We decompose the Juttner and Maxwell-Boltzman functions (in the beta representation $\beta=\mathrm{V} / c$ ) into the Fourier cosine series and note that Maxwell-Boltzman and Juttner functions are approximated with a high precision by six terms of this decomposition (these terms are $1 / 2, \cos (\pi \beta), \cos (2 \pi \beta), \cos (3 \pi \beta)$, $\cos (4 \pi \beta)$, and $\cos (5 \pi \beta))$. For a plasma with a temperature of $15.3 \mathrm{keV}$ (which corresponds to $\Theta=k_{\mathrm{B}} T_{\mathrm{e}} /\left(m_{\mathrm{e}} c\right)=0.03$ ), 


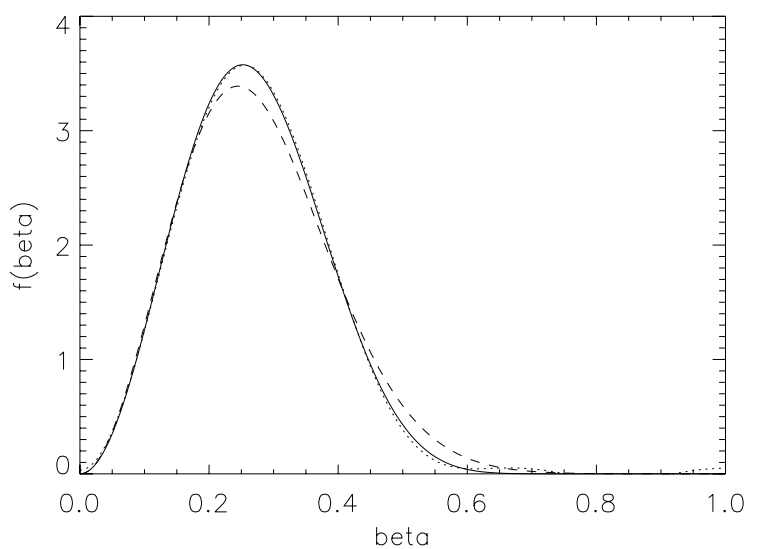

Fig. 4. Relativistic and non-relativistic DFs, and the approximation to a Juttner distribution obtained by means of six terms of Fourier series shown by the solid, dashed, and dotted lines, respectively.

the decomposition coefficients of a Juttner function approximately equal $2.000,1.256,-0.172,-0.954,-0.786,-0.310$ for the terms $1 / 2, \cos (\pi \beta), \cos (2 \pi \beta), \cos (3 \pi \beta), \cos (4 \pi \beta)$, and $\cos (5 \pi \beta)$, respectively. For a plasma with a temperature of $15.3 \mathrm{keV}$, the decomposition coefficients of a Maxwell-Boltzman function approximately equal $2.000,1.214,-0.204,-0.878$, $-0.699,-0.316$ for the terms $1 / 2, \cos (\pi \beta), \cos (2 \pi \beta), \cos (3 \pi \beta)$, $\cos (4 \pi \beta)$, and $\cos (5 \pi \beta)$, respectively. Note that the coefficient at the first term equals 2, because DFs are normalized. Juttner and Maxwell-Boltzman functions for a temperature $15.3 \mathrm{keV}$ are shown in Fig. 4 by solid and dashed lines, respectively. The approximation to a Juttner distribution obtained by means of six terms of Fourier series is shown in Fig. 4 by a dotted line. Figure 4 shows that six terms of the Fourier series is sufficient to describe the main features of DFs: an increase of the Juttner DF in the range $\beta=[0.2,0.4]$ and a decrease of the DF in the range $\beta=[0.4,0.6]$ with respect to the Maxwell-Boltzman DF, which arise from relativistic corrections.

To show how a velocity DF of electrons can be derived from multi-frequency SZ observations, we write Eq. (3) by using the generalized spectral function $G\left(x, T_{\mathrm{e}}\right)$ introduced by Prokhorov et al. (2010b) given by the expression

$G\left(x, f_{\mathrm{e}}\right)=\int \frac{P_{1}\left[s, f_{\mathrm{e}}\right]}{\Theta\left(T_{\mathrm{e}}\right)}\left(\frac{x^{3} \mathrm{e}^{-3 s}}{\exp \left(x \mathrm{e}^{-s}\right)-1}-\frac{x^{3}}{\mathrm{e}^{x}-1}\right) \mathrm{d} s$.

Thus, the CMB intensity change caused by the SZ effect is $\Delta I=I_{0} \tau\left(k_{\mathrm{B}} T_{\mathrm{e}} /\left(m_{\mathrm{e}} c^{2}\right)\right) \times G\left(x, T_{\mathrm{e}}\right)$. Note that in this notation, the spectral function $g_{\mathrm{nr}}(x)$ is changed to the generalized spectral function $G\left(x, T_{\mathrm{e}}\right)$, which explicitly depends on the electron temperature.

The CMB intensity change caused by the SZ effect for a DF represented by six terms of Fourier cosine series is

$\Delta I=I_{0} \tau \sum_{\mathrm{k}=0}^{5} A_{\mathrm{k}} \int \mathrm{d} s P_{1}\left[s,-1 / 2 \delta_{\mathrm{k}}+\cos (\pi k \beta)\right] K(s, x)$,

where $\delta_{\mathrm{k}}$ is the Kronecker delta function, $A_{\mathrm{k}}$ are the Fourier coefficients given by

$A_{\mathrm{k}}=2 \int_{0}^{1} f_{\mathrm{e}}(\beta) \cos (\pi k x) \mathrm{d} x$

for $k=0,1,2,3,4,5$; and

$K(s, x)=\left(\frac{x^{3} \mathrm{e}^{-3 s}}{\exp \left(x \mathrm{e}^{-s}\right)-1}-\frac{x^{3}}{\mathrm{e}^{x}-1}\right)$.
Multiplying the left-hand and right-hand sides of Eq. (8) by $1 / \Theta\left(T_{\mathrm{e}}\right)$, we rewrite Eq. (8) in terms of the generalized spectral function $G\left(x, T_{\mathrm{e}}\right)$ in the form

$\frac{\Delta I}{I_{0} \tau \Theta\left(T_{\mathrm{e}}\right)}=\sum_{\mathrm{k}=0}^{5} A_{\mathrm{k}} G\left(x,-1 / 2 \delta_{\mathrm{k}}+\cos (\pi k \beta)\right)$.

Since $A_{0}=2$ because of the DF normalization, we need to find only five Fourier coefficients. Using multi-frequency measurements of the SZ effect, we can find a solution for the linear system of equations

$\frac{\Delta I\left(x_{\lambda}\right)}{I_{0} \tau \Theta\left(T_{\mathrm{e}}\right)}=\sum_{\mathrm{k}=0}^{5} A_{\mathrm{k}} G\left(x_{\lambda},-1 / 2 \delta_{\mathrm{k}}+\cos (\pi k \beta)\right)$

in order to derive the Fourier coefficients $A_{\mathrm{k}}$, for $\lambda=1,2,3,4,5$.

Note that the Comptonization parameter should be independently derived from a precise SZ intensity measurement at a frequency of $v=255 \mathrm{GHz}(x=4.5)$, since the SZ intensities for Juttner and Maxwell-Boltzman DFs have the same value at this frequency (see Fig. 1).

To make the system of linear equations well-conditioned, we constrain our analysis of DFs by considering the functions for which $f_{\mathrm{e}}(\beta=0)=0$. This choice is consistent with both Juttner and Maxwell-Boltzman DFs. In this case, taking into account that $A_{0}=2$, the system of linear equations (12) can be written as

$$
\begin{gathered}
\sum_{\mathrm{k}=1}^{5} A_{\mathrm{k}}\left(G\left(x_{\lambda},-1 / 2 \delta_{\mathrm{k}}+\cos (\pi k \beta)\right)-g_{\mathrm{nr}}\left(x_{\lambda}\right)\right)= \\
g_{\mathrm{nr}}\left(x_{\lambda}\right)+\frac{\Delta I\left(x_{\lambda}\right)}{I_{0} \tau \Theta\left(T_{\mathrm{e}}\right)}-\int \frac{P_{1}[s, 1]}{\Theta\left(T_{\mathrm{e}}\right)} K\left(s, x_{\lambda}\right) \mathrm{d} s
\end{gathered}
$$

To check if this system of linear equations is well-conditioned, we study the properties of the matrix $M_{\lambda \mathrm{k}}$, which is given by

$M_{\lambda k}=G\left(x_{\lambda},-1 / 2 \delta_{\mathrm{k}}+\cos (\pi k \beta)\right)-g_{\mathrm{nr}}\left(x_{\lambda}\right)$

for different samples of frequencies.

To find a suitable sample of frequencies for deriving the electron DF, let us consider the following samples: the first sample is in the low-frequency range including four frequencies $v=100$, $120,140,160 \mathrm{GHz}$ considered by Colafrancesco \& Marchegiani (2010); the second sample including four frequencies in the frequency range $300-400 \mathrm{GHz}$ considered by Colafrancesco \& Marchegiani (2010), $v=300,320,340,360 \mathrm{GHz}$; the third sample including low and high frequencies $v=100,200,300$, $400 \mathrm{GHz}$; and the fourth sample including more higher frequencies $v=375,600,700,857 \mathrm{GHz}$.

The first detection of the Sunyaev-Zel'dovich effect increment at such high frequencies where the relativistic corrections of the SZ effect are relevant has been obtained with HERSCHEL-SPIRE for the Bullet cluster (Zemcov et al. 2010).

To find the frequency sample for which the electron DF can be optimally derived, we calculate the condition numbers for the matrixes of $M_{\lambda k}$ and the inverse matrixes of $M_{\mathrm{k} \lambda}^{-1}$. Since these samples have only four frequencies, the equation obtained from the condition of $f_{\mathrm{e}}(\beta=0)=0$ is used $A_{1}+A_{2}+A_{3}+A_{4}+A_{5}=-1$ (the normalization condition $A_{0}=2$ is taken into account) to form the matrix $5 \times 5$, we choose the first row of these matrixes equaled to $M_{1 k}=(1,1,1,1,1)$. The condition numbers based on the $L_{2}$ norm of these matrixes approximately equal to $4.4 \times 10^{4}$, $2.1 \times 10^{5}, 1.6 \times 10^{3}$, and 323.8 for the $1 \mathrm{st}, 2 \mathrm{nd}, 3 \mathrm{rd}$, and $4 \mathrm{th}$ samples, respectively. Since the condition number of the matrix 
corresponding to the fourth frequency sample is the smallest, we conclude that the best sample to determine DFs by means of the SZ effect is the fourth sample, which includes high frequencies. The worst sample is the second sample, which is in the frequency range $300-400 \mathrm{GHz}$, since the matrix $M_{\lambda \mathrm{k}(2)}$ is ill-conditioned.

To clarify our method of the DF analysis, we calculate the inverse matrixes

$$
\begin{aligned}
M_{\mathrm{k} \lambda(1)}^{-1}= & \left(\begin{array}{ccccc}
-0.86 & 0.15 & -0.59 & 1.59 & -1.05 \\
-3.11 & -19.35 & 18.60 & 5.30 & -6.74 \\
-5.28 & 190.19 & -522.48 & 499.98 & -164.28 \\
2.00 & 159.65 & -358.39 & 294.83 & -89.09 \\
8.25 & -330.66 & 862.87 & -801.71 & 261.18
\end{array}\right) \quad(15) \\
M_{\mathrm{k} \lambda(2)}^{-1}= & \left(\begin{array}{ccccc}
-0.89 & -6.89 & 25.02 & -28.79 & 10.69 \\
-3.83 & 42.01 & -112.45 & 97.69 & -27.23 \\
-6.68 & 808.77 & -2462.02 & 2509.77 & -856.15 \\
2.79 & 331.94 & -1046.14 & 1104.08 & -390.21 \\
9.63 & -1175.84 & 3595.60 & -3682.75 & 1262.90
\end{array}\right)
\end{aligned}
$$

$M_{\mathrm{k} \lambda(3)}^{-1}=\left(\begin{array}{ccccc}-0.88 & 0.28 & -0.14 & -0.03 & 0.02 \\ -3.75 & -0.90 & 1.76 & -2.14 & 1.01 \\ -6.76 & -22.04 & 25.24 & -11.68 & 1.06 \\ 2.57 & -9.60 & -1.86 & -1.86 & -1.71 \\ 9.82 & 32.25 & -36.24 & 15.70 & -0.38\end{array}\right)$

$M_{\mathrm{k} \lambda(4)}^{-1}=\left(\begin{array}{ccccc}-0.21 & 0.31 & -2.18 & 2.79 & -0.96 \\ -2.36 & -0.06 & -2.43 & 3.95 & -1.61 \\ -2.79 & -0.38 & -2.06 & 5.24 & -3.19 \\ 0.86 & -1.00 & 6.94 & -9.18 & 2.95 \\ 5.49 & 1.12 & -0.28 & -2.79 & 2.81\end{array}\right)$

The experimental uncertainties in the SZ intensity measurements constrain the ability to derive DFs of electrons. The uncertainties in the determination of the Fourier coefficients of $A_{k}$ are given by

$\delta A_{j}=\sum_{\lambda} M_{\mathrm{j} \lambda}^{-1} \frac{\delta\left(\Delta I\left(x_{\lambda}\right) / I_{0}\right)}{\tau \Theta\left(T_{\mathrm{e}}\right)}$

and can be written as

$\delta A_{j}=\sum_{n} M_{\mathrm{jn}}^{-1} \sum_{\lambda} \tilde{G}_{\mathrm{n} \lambda} V_{\lambda}$

where

$V_{\lambda}=\left(\frac{\delta(\Delta I(x))}{\Delta I(x)}\right)_{x=x_{\lambda}}$

for $\lambda=2,3,4,5$ and $V_{1}=0$ (since the first term determined from the condition $f(\beta=0)=0)$, and

$\tilde{G}_{\mathrm{n} \lambda}=\left(\begin{array}{ccccc}0 & 0 & 0 & 0 & 0 \\ 0 & G\left(x_{1}, T_{\mathrm{e}}\right) & 0 & 0 & 0 \\ 0 & 0 & G\left(x_{2}, T_{\mathrm{e}}\right) & 0 & 0 \\ 0 & 0 & 0 & G\left(x_{3}, T_{\mathrm{e}}\right) & 0 \\ 0 & 0 & 0 & 0 & G\left(x_{4}, T_{\mathrm{e}}\right)\end{array}\right)$

where $x_{1}, x_{2}, x_{3}, x_{4}$ correspond to the considered frequencies ( $v=375,600,700,857 \mathrm{GHz}$ for the fourth sample) and the generalized spectral functions $G\left(x_{\lambda}, T_{\mathrm{e}}\right)$ are calculated for a Juttner DF (this is because we study the ability of multi-frequency SZ observations to distinguish a Juttner DF from other DFs, which are slightly different from a Juttner DF).

Equation (20) can be written as

$\delta A_{j}=\sum_{\lambda} W_{\mathrm{j} \lambda} V_{\lambda}$

where

$W_{\mathrm{j} \lambda}=\sum_{n} M_{\mathrm{jn}}^{-1} \tilde{G}_{\mathrm{n} \lambda}$

The matrixes $W_{\mathrm{j} \lambda}$ are shown below for the samples of frequencies $v=375,600,700,857 \mathrm{GHz}$ and $v=300,320,340$, $360 \mathrm{GHz}$ for a comparison.

$W_{\mathrm{j} \lambda(4)}=\left(\begin{array}{ccccc}0 & 1.75 & -6.27 & 4.61 & -0.62 \\ 0 & -0.33 & -6.99 & 6.53 & -1.03 \\ 0 & -2.10 & -5.92 & 8.67 & -2.04 \\ 0 & -5.54 & 19.99 & -15.19 & 1.89 \\ 0 & 6.22 & -0.80 & -4.62 & 1.80\end{array}\right)$

$W_{\mathrm{j} \lambda(2)}=\left(\begin{array}{ccccc}0 & -27.07 & 115.26 & -146.87 & 57.94 \\ 0 & 165.02 & -517.97 & 498.23 & -147.59 \\ 0 & 3176.87 & -11340.09 & 12799.83 & -4640.34 \\ 0 & 1303.88 & 4818.54 & 5630.83 & -2114.94 \\ 0 & -4618.70 & 16561.34 & -18782.03 & 6844.92\end{array}\right)$. (26

Since the absolute values of the elements of $\tilde{W}_{\mathrm{j} \lambda(4)}$ are much smaller than those of $\tilde{W}_{\mathrm{j} \lambda(2)}$, we justify our previous conclusion that the fourth sample is more suitable for the SZ analysis of electron DF than the second sample, since in the case of the fourth sample to derive a DF of electrons the allowable uncertainties in the SZ intensities are about three orders of magnitude larger (see Eq. (23)). We also checked that the fourth sample is the best amongst the all samples considered in this paper in order to study DFs.

To study the ability to derive DFs from SZ intensity measurements, we calculate the differences between the corresponding Fourier coefficients for Juttner and Maxwell-Boltzman DFs, which are $\left|A_{1, \mathrm{~J}}-A_{1, \mathrm{MB}}\right|=0.042,\left|A_{2, \mathrm{~J}}-A_{2, \mathrm{MB}}\right|=0.032$, $\left|A_{3, \mathrm{~J}}-A_{3, \mathrm{MB}}\right|=0.076,\left|A_{4, \mathrm{~J}}-A_{4, \mathrm{MB}}\right|=0.087$, and $\left|A_{5, \mathrm{~J}}-A_{5, \mathrm{MB}}\right|=$ 0.006 (see the previous section).

Note that the difference $\left|A_{5, \mathrm{~J}}-A_{5, \mathrm{MB}}\right|=0.006$ is very small and it will be difficult to observe this difference by the SZ measurements. However, we checked that this difference has a negligible impact on the precision of the approximation of a Juttner DF obtained by means of Fourier series because of its small value.

For the sake of illustration, we assume that the relative SZ intensity uncertainty in measurements $\delta\left(\Delta I\left(x_{\lambda}\right)\right) / \Delta I_{x_{\lambda}}$ does not depend on the frequency and equals $\xi$.

From Eqs. (23) and (25) we find that the uncertainty in SZ intensity measurements to distinguish the values of the Fourier coefficients for Juttner and Maxwell-Boltzman DFs should be less than $\xi=0.32 \%$ to derive the $A_{1}$ value, $\xi=0.22 \%$ to derive the $A_{2}$ value, $\xi=0.41 \%$ to derive the $A_{3}$ value, and $\xi=0.20 \%$ to derive the $A_{4}$ value.

To check the result that an electron DF can be derived by means of the SZ effect if uncertainties of SZ observational data are less than $0.2 \%$, we performed Monte-Carlo simulations of SZ observations (calculated in the relativistic correct formalism and using a Juttner DF) with the SZ intensity uncertainty of $0.1 \%$ at the frequencies included in the fourth sample. Using the SZ spectra obtained from the Monte-Carlo simulations, we 


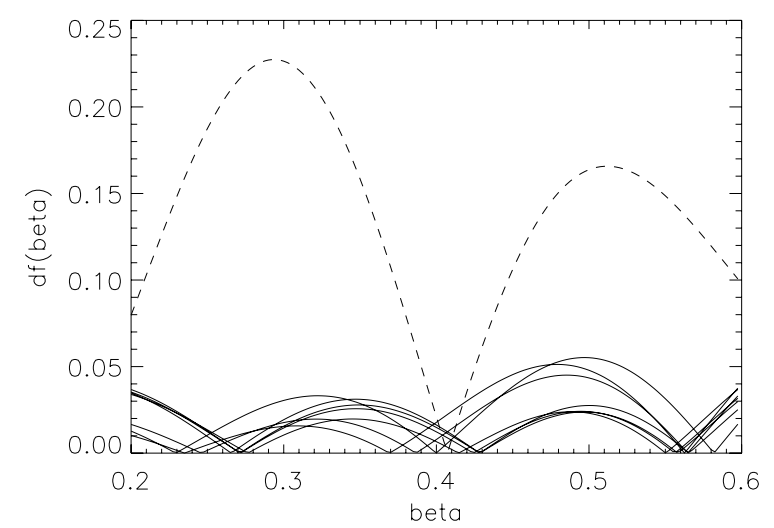

Fig. 5. Absolute difference between the Juttner and approximate DFs for a temperature of $15.3 \mathrm{keV}$ (solid lines) compared with the absolute difference between the Juttner and Maxwell-Boltzman DFs (dashed line).

solve the system of equations (13) to find the Fourier coefficients $A_{\mathrm{k}}$ and the approximate functions to a Juttner DF, which has been initially used to produce the CMB intensity changes caused by the SZ effect in Monte-Carlo simulations. The absolute value of the difference between the Juttner and Maxwell-Boltzman DFs for a temperature of $15.3 \mathrm{keV}$ in the range of $\beta=[0.2,0.6]$ is shown in Fig. 5 by a dashed line. This range of $\beta$ contains the main features of these DFs: an increase of the Juttner DF in the range $\beta=[0.2,0.4]$ and a decrease of the Juttner DF in the range $\beta=[0.4,0.6]$ with respect to the Maxwell-Boltzman DF. The absolute difference between the Juttner DF for a temperature of $15.3 \mathrm{keV}$ and the approximate functions to the Juttner DF obtained by the Monte-Carlo simulations are shown in Fig. 5 by solid lines. Figure 5 shows that the approximate DFs are close to a Juttner DF and it is possible to distinguish the Juttner and Maxwell-Boltzman DFs, if the SZ intensities are measured with an uncertainty of $0.1 \%$ (see Sect. 7 below).

We conclude that the SZ effect provides us with an interesting method to study electron DFs in massive galaxy clusters that contain hot plasmas with temperatures $(\simeq 15 \mathrm{keV})$.

\section{Relaxation of a system of electrons to equilibrium distributions}

To justify the use of a Juttner distribution function as an appropriate approximation to the universal (equilibrium) electron distribution in hot merging clusters, we calculate the electron equilibration time and compare this time with the merging time scale. We consider here the relaxation to equilibrium distributions in the framework of both the non-relativistic and relativistic theories.

We solve numerically the time-dependent Fokker-Planck equation for the evolution of an isotropic system of electrons with Coulomb interactions and with an initially Gaussian momentum distribution. The Fokker-Planck equation coefficients are taken from Liang et al. (2002) and Dogiel et al. (2007). The initial Gaussian momentum distributions are chosen so that the number density normalization and the total energy are consistent with those of the equilibrium distributions. Note that both the Fokker-Planck coefficients (see Dogiel et al. 2007) are proportional to $m_{\mathrm{e}} c^{2} /\left(k_{\mathrm{B}} T_{\mathrm{e}}\right)$ and, therefore, the value of the characteristic frequency (which determines the rate of relaxation) is given by the inverse Spitzer collision time (see Eq. (18) of Liang et al. 2002). We use here the dimensionless time $\tau$ in units of the Spitzer collision time. For an electron population with number

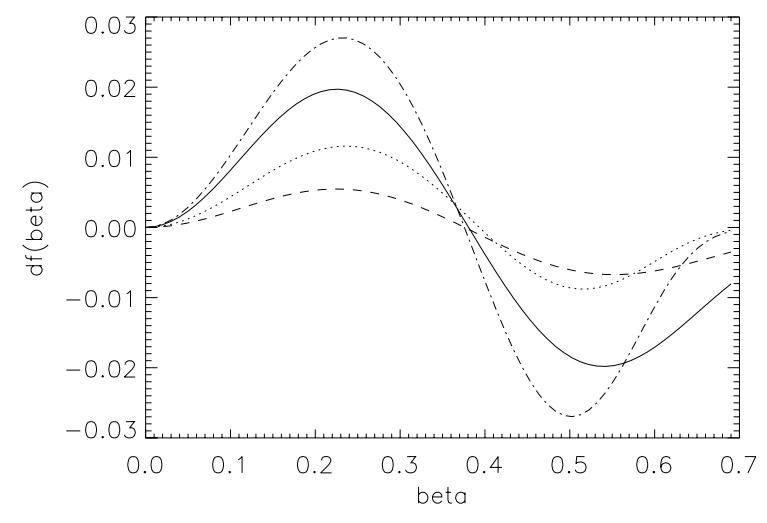

Fig. 6. Difference between the electron distribution function and the Maxwell-Boltzman DF at time $\tau=40$ (solid) and 60 (dashed) is shown as a function of $\beta=v / c$. The analogous difference between the electron distribution function and the Juttner DF at time $\tau=40$ (dash-dotted) and 60 (dotted) is also shown.

density of $n=10^{-3} \mathrm{~cm}^{-3}$ and temperature $T_{\mathrm{e}}=15.3 \mathrm{keV}$, the Spitzer collision time equals $1.3 \times 10^{5} \mathrm{yr}$.

The results of our numerical analysis of relaxation of a system of electrons to equilibrium distributions are plotted in Fig. 6.

The solid and dashed curves in Fig. 6 show the difference between the electron distribution function, derived in the framework of the non-relativistic theory, at times $\tau=40$ and 60, respectively, and the reference Maxwell-Boltzman DF. Since these differences are significantly smaller than the difference between the Juttner and Maxwell-Boltzman DFs shown in Fig. 5, the electron DFs at time $\tau=40$ and 60 can be considered as good approximations to the Maxwell-Boltzman DF.

The dash-dotted and dotted curves in Fig. 6 show the difference between the electron distribution function, derived in the framework of the relativistic theory, at times $\tau=40$ and 60 , respectively, and the reference Juttner DF. These differences are again significantly smaller than the difference between the Juttner and Maxwell-Boltzman DFs shown in Fig. 5 and, therefore, the electron DFs at a time of $\tau=40$ and 60 can be considered as good approximation to the Juttner DF.

We stress that the qualitative estimate of the time required to fill the Maxwell tail to the velocity of $0.7 c$ in the non-relativistic theory (see, e.g., MacDonald \& Rosenbluth 1957) is twenty five Spitzer collision times and is in rough agreement with our numerical analysis.

Since sixty Spitzer collision times correspond to $\approx 8.1 \times$ $10^{6} \mathrm{yrs}$ for the number density and temperature values mentioned above, this time scale is a tiny fraction of the Hubble time. The merging time scale of a merging cluster equaled to $\simeq 10^{8}-10^{9}$ yrs (see, e.g., Prokhorov \& Durret 2007; Akahori \& Yoshikawa 2010) is longer than sixty Spitzer collision times. Thus, we conclude that the Juttner distribution function is an appropriate approximation to the universal (equilibrium) electron distribution in hot merged clusters.

We estimate the gas density at which the time-dependent DF relaxing to a Maxwell-Boltzman DF is indistinguishable from a Juttner DF. The time, at which the difference between the time-dependent DF and the Maxwell-Boltzman DF is $\approx 0.2$ and at which the time-dependent DF is close to a Juttner DF (see Fig. 5), corresponds to five Spitzer times or $\simeq 6.5 \times 10^{5}$ yrs for an electron number density of $10^{-3} \mathrm{~cm}^{-3}$. Using a merging time scale of $\gtrsim 10^{8}$ yrs, we found that the value of the critical number density $\lesssim 6.5 \times 10^{-6} \mathrm{~cm}^{-3}$. This electron number density is much smaller than the average gas density in galaxy clusters 
(see e.g. Sarazin 1986) and, therefore, it is possible to distinguish a Juttner DF from a Maxwell-Boltzman DF in galaxy clusters by the method proposed in Sect. 3.

\section{DFs in galaxy clusters with hard X-ray spectral tails}

Galaxy clusters with a hard X-ray excess are promising targets to test electron DFs by means of multi-frequency SZ observations. This is because these clusters show evidence of either a very high temperature plasma or a quasi-thermal emission tail due to MHD acceleration mechanisms in the cluster atmosphere.

Petrosian (2001) estimated the yield in non-thermal bremsstrahlung photons and found that a large amount of the energy of the non-thermal electrons is transferred to the background plasma, so that the ICM should be heated to above its observable temperature within $10 \mathrm{Myr}$. However, it was shown by Liang et al. (2002) and Dogiel et al. (2007) that a quasithermal electron population might overcome this difficulty by means of a higher radiative efficiency (and therefore a longer overheating time, but see Petrosian \& East 2008). Wolfe \& Melia (2008) also considered a quasi-thermal electron distribution when fitting hard $\mathrm{X}$-ray emission, but rather than requiring a second-order Fermi acceleration to produce quasi-thermal electrons, they assumed that quasi-thermal electrons are produced by collisions with non-thermal protons.

A study of the influence of suprathermal electrons on the SZ effect was made for the Coma and Abell 2199 clusters by Blasi et al. (2000), Shimon \& Rephaeli (2002), and Colafrancesco et al. (2009). An alternative probe to study the electron distribution in galaxy clusters, namely the flux ratio of the emission lines caused by $\mathrm{FeK} \alpha$ transitions (FeXXV and FeXXVI) was proposed by Prokhorov et al. (2009). This flux ratio is very sensitive to the population of electrons with energies higher than the ionization potential of a FeXXV ion (which is $8.8 \mathrm{keV}$ ). Kaastra et al. (2009) demonstrated that the relative intensities of the satellite lines are sensitive to the presence of suprathermal electrons in galaxy clusters. Prokhorov (2009) studies the influence of high-energy electron populations on metal abundance estimates in galaxy clusters and shows that the effect of highenergy particles can be significant. However, the mentioned approaches do not allow us to derive electron DFs in galaxy clusters with a hard X-ray excess.

In the section, we propose an approach to derive the electron DF and demonstrate that multi-frequency SZ observations are promising for this purpose.

Evidence for non-thermal X-ray emission from the Bullet cluster reported by Petrosian et al. (2006) and Million \& Allen (2009) suggests a possible high-energy subrelativistic electron component if these X-ray spectra are interpreted in terms of bremsstrahlung emission. Therefore, the Bullet cluster is an interesting target to test electron DFs. We calculate the SZ intensity spectra for the Bullet cluster for DFs with and without the second electron population proposed by Petrosian et al. (2006). These SZ spectra are shown in Fig. 7. Here we assume that the second thermal electron population with $k_{\mathrm{B}} T_{\mathrm{e}, 2}=50 \mathrm{keV}$ mimics the presence of quasi-thermal electrons in the DF and that the second electron population is $5 \%$ of the first thermal electron population. This fraction of high-energy subrelativistic electrons is consistent with that derived by Petrosian et al. (2006). For the sake of comparison with the results obtained in the previous section, we assume that the temperature of the first thermal component equals $15.3 \mathrm{keV}$, which agrees with the temperature

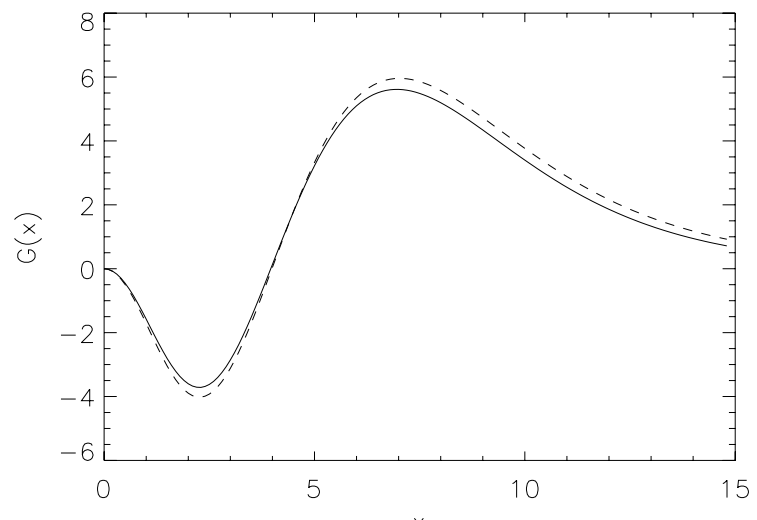

Fig. 7. SZ intensity spectra $G(x)=\Delta I(x) /\left(I_{0} y\right)$ for the cluster with the hard X-ray tail for DFs with and without the second electron population shown by the dashed and solid lines, respectively.

values of $14.5_{-1.7}^{+2.0} \mathrm{keV}$ and $14.8_{-1.2}^{+1.7} \mathrm{keV}$ derived by using both ASCA and ROSAT (Liang et al. 2000) and by using Chandra data (Markevitch et al. 2002), respectively. Note that the total DF is normalized. The Comptonization parameter should be independently derived from a precise SZ intensity measurement at a frequency $v=250 \mathrm{GHz}(x=4.4)$, since the SZ intensities have the same value at this frequency for normalized Juttner DFs with and without the second electron population (see Fig. 7).

For a plasma with these parameters, the decomposition coefficients of the DF with the second electron component approximately equal to $2.000,1.207,-0.220,-0.917,-0.741,-0.298$ for the terms $1 / 2, \cos (\pi \beta), \cos (2 \pi \beta), \cos (3 \pi \beta), \cos (4 \pi \beta)$, and $\cos (5 \pi \beta)$, respectively. To analyze the DF, we use the method of multi-frequency SZ observations at the frequencies of $v=375$, $600,700,857 \mathrm{GHz}$ described in the previous section. To study our ability to derive DFs from SZ intensity measurements, we calculate the differences between the corresponding Fourier coefficients for normalized Juttner DFs with and without the second electron component, which are $\left|\Delta A_{1}\right|=0.049,\left|\Delta A_{2}\right|=$ $0.047,\left|\Delta A_{3}\right|=0.037,\left|\Delta A_{4}\right|=0.045$, and $\left|\Delta A_{5}\right|=0.012$. Note that the difference $\left|\Delta A_{5}\right|=0.012$ is small and it will be difficult to observe this difference by the SZ measurements. However, we have checked that this difference has a negligible impact on the precision of the approximation of the DF obtained by means of Fourier series because of its small values.

From Eqs. (23) and (25) we find that the uncertainty in SZ intensity measurements to distinguish the values of the Fourier coefficients for normalized Juttner DFs with and without the second electron component should be less than: $\xi=0.37 \%$ to derive the $A_{1}$ value, $\xi=0.32 \%$ to derive the $A_{2}$ value, $\xi=0.20 \%$ to derive the $A_{3}$ value, and $\xi=0.1 \%$ to derive the $A_{4}$ value. Therefore, the electron DF can be derived by means of the SZ effect if uncertainties of SZ observational data are less than $0.1 \%$.

To show that multi-frequency SZ observations allow us to derive the Juttner DF with the second electron component, we calculate the SZ intensity (Eq. (3)) at the frequencies of $v=375$, $600,700,857 \mathrm{GHz}$ assuming this DF. We find the decomposition coefficients from Eq. (13) by means of the simulated SZ observations and show the obtained approximation to the Juttner distribution with the second electron population in Fig. 8 by a dotted line in the beta range of $\beta=[0,0.7]$. The normalized Juttner DFs with and without the second electron population are also shown in Fig. 8 by dashed and solid lines, respectively.

Since the approximate function obtained by means of the simulated SZ observations coincides precisely with the 


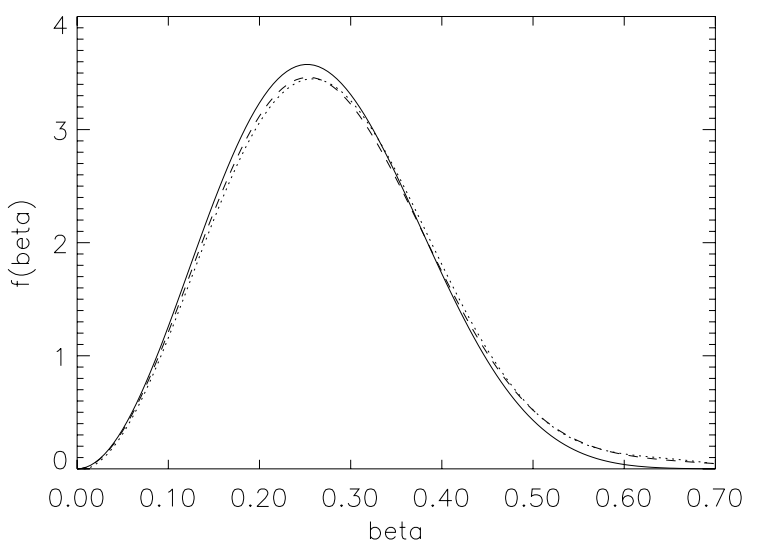

Fig. 8. Normalized Juttner DFs with and without the second electron population, and the approximation to a Juttner distribution with the second electron population obtained by means of the simulated SZ observations shown by the solid, dashed, and dotted lines, respectively.

normalized Juttner DF with the second electron population, we conclude that precise observations of the SZ effect can allow to derive the electron DFs in galaxy clusters with a hard X-ray excess if electron DFs are different from a Juttner DF.

\section{SZ intensity maps for the simulated merging galaxy cluster}

In the previous sections, we showed that high-frequency spectral SZ observations at frequencies $v=375 \mathrm{GHz}, 600 \mathrm{GHz}$, $700 \mathrm{GHz}$, and $857 \mathrm{GHz}$ provide us with a method to derive DFs of electrons in galaxy clusters.

Here we study the intensity maps of hot clusters at these frequencies. Note that all the SZ intensity maps derived in the Kompaneets approximation have the same spatial morphology. Since the shape of the SZ effect is most sensitive to an electron DF at frequencies $v=375 \mathrm{GHz}, 600 \mathrm{GHz}, 700 \mathrm{GHz}$, and $857 \mathrm{GHz}$ (see Sects. 3 and 4), the spatial SZ intensity maps, which are derived in the Wright formalism, at these frequencies should have different spatial morphology.

In this section, we show that the spatial morphologies of the SZ intensity maps are different at frequencies $v=375 \mathrm{GHz}$, $600 \mathrm{GHz}, 700 \mathrm{GHz}$, and $857 \mathrm{GHz}$. To this aim we use the 3D numerical hydrodynamic simulations of a merging hot galaxy cluster presented in Akahori \& Yoshikawa (2010). These authors considered a head-on encounter of two free-falling galaxy clusters from the turn-around radius (for a review, see Sarazin 2002). We assume in the simulation that the two galaxy clusters have equal virial mass of $M_{\mathrm{vir}}=8 \times 10^{14} M_{\odot}$, and the impact parameter is zero (for details on the simulation, see Akahori \& Yoshikawa 2010).

To produce the SZ intensity maps at frequencies $v=$ $375 \mathrm{GHz}, 600 \mathrm{GHz}, 700 \mathrm{GHz}$, and $857 \mathrm{GHz}$ we use the 3D density and temperature maps (see Figs. 2 and 3 from Akahori \& Yoshikawa 2010) for the simulated merging galaxy cluster at a time of $t=0.5 \mathrm{Gyr}$, where $t=0 \mathrm{Gyr}$ corresponds to the time of the closest approach of the centers of the dark matter halos. We calculated the SZ intensity maps using the Wright formalism and the approach described in Prokhorov et al. (2010a,b). The intensity maps of the SZ effect at these frequencies derived from the simulated maps of the gas density and temperature are shown in Figs. 9-12, respectively.

The morphologies of the SZ intensity simulated maps at $v=$ $375 \mathrm{GHz}, 600 \mathrm{GHz}, 700 \mathrm{GHz}$, and $857 \mathrm{GHz}$ are clearly different.

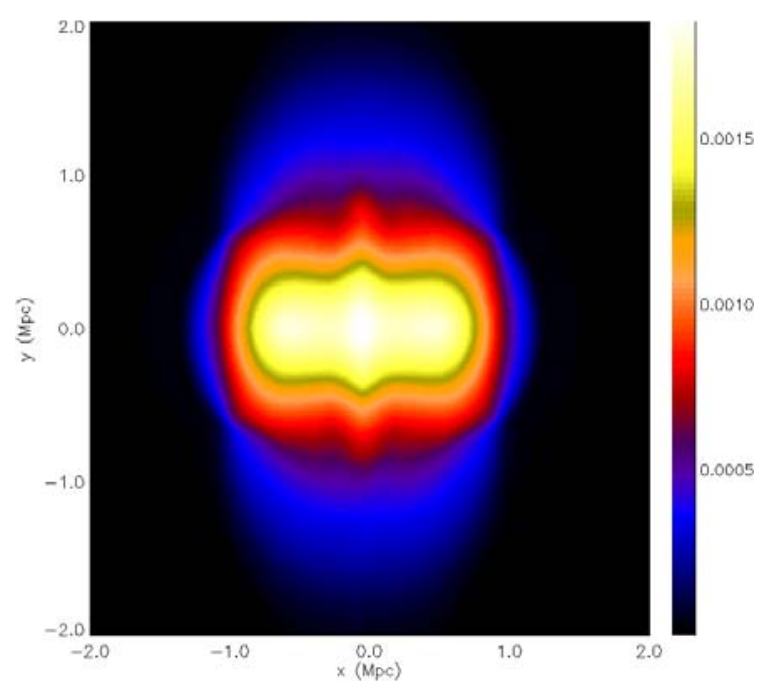

Fig. 9. Intensity map $I / I_{0}$ of the $\mathrm{SZ}$ effect at a frequency $375 \mathrm{GHz}$ derived from the numerical simulation in the framework of the Wright formalism

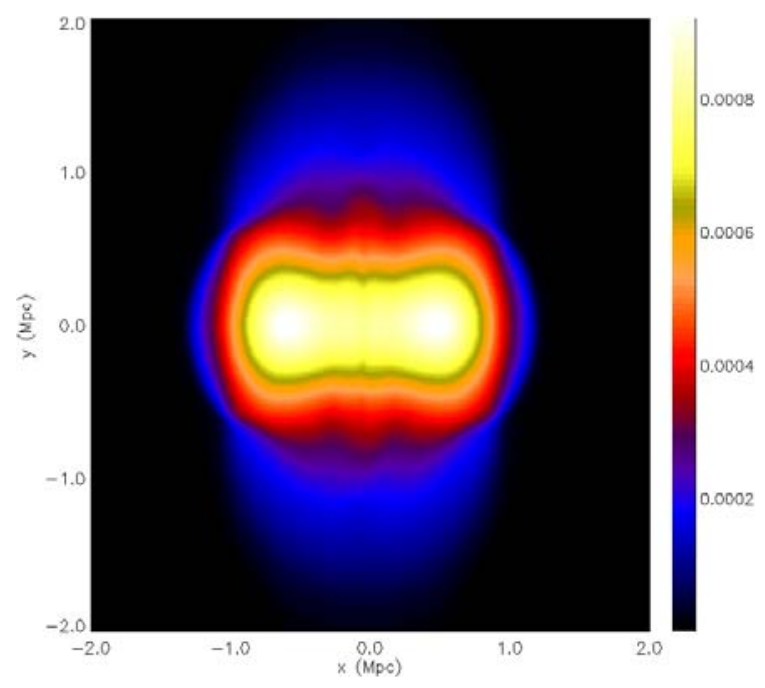

Fig. 10. Same as Fig. 9 but for a frequency of $600 \mathrm{GHz}$.

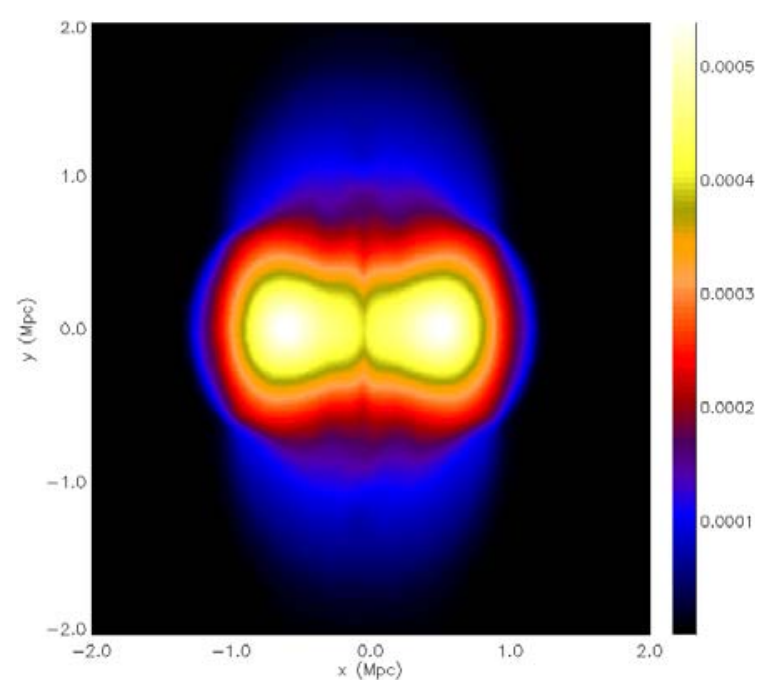

Fig. 11. Same as Fig. 9 but for a frequency of $700 \mathrm{GHz}$.

This is because the SZ effect from hot galaxy clusters at high frequencies is sensitive to the relativistic effects in their electron 


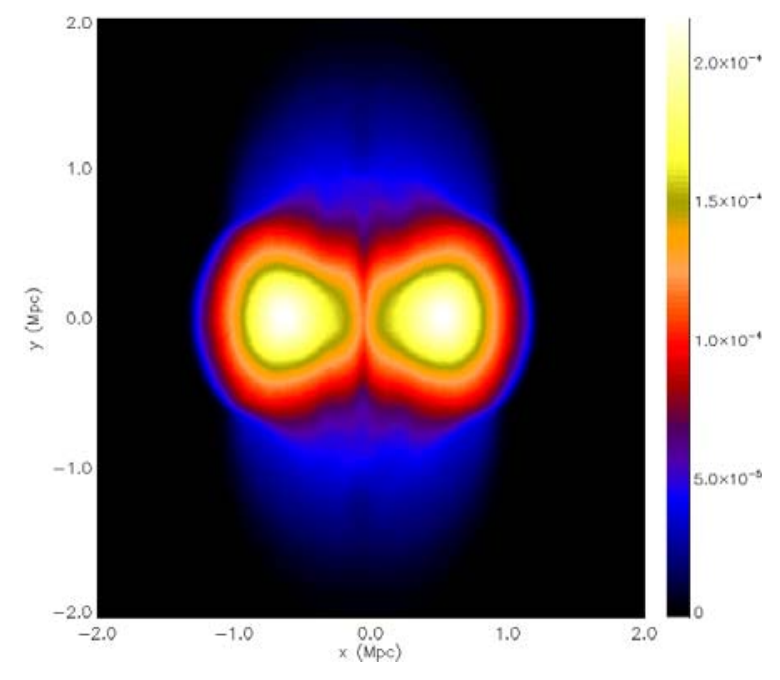

Fig. 12. Same as Fig. 9 but for a frequency of $857 \mathrm{GHz}$.

DF and cannot be described in the framework of the Kompaneets approximation. Note that the morphologies of the SZ intensity simulated maps at frequencies $v=128 \mathrm{GHz}$ and $369 \mathrm{GHz}$ in Prokhorov et al. (2010a) are instead more similar because the SZ effect from cool galaxy clusters is less sensitive to the relativistic effects.

We find that the SZ intensity simulated map at frequency of $375 \mathrm{GHz}$ is similar to the SZ intensity simulated map derived in the Kompaneets approximation, while the SZ intensity simulated map at a frequency of $857 \mathrm{GHz}$ is similar to the SZ intensity simulated map at a frequency of $217 \mathrm{GHz}$ where the SZ effect in the framework of the Kompaneets approximation is zero. The contribution of relativistic corrections to the SZ signal at frequency of $857 \mathrm{GHz}$ dominates over that derived in the Kompaneets approximation.

We find that the maximum of the SZ intensity increment at a frequency of $375 \mathrm{GHz}$ (see Fig. 9) is at the center of this map, while the maximum of the SZ intensity increment at a frequency of $857 \mathrm{GHz}$ (see Fig. 12) is in the post-shock regions (see the Mach number distribution of ICM in Fig. 2 of Akahori \& Yoshikawa 2010). This is because the gas temperature in the post-shock regions is the highest and SZ relativistic corrections are therefore the most significant in these regions.

Comparing Fig. 9 with 12 shows that the SZ intensity maps at low and high frequencies look different for a hot merging galaxy cluster and, therefore, multi-frequency SZ observations with a high spatial resolution are necessary to demonstrate the different morphologies of SZ intensity maps at low and high frequencies. We conclude that measurements of the SZ intensity maps at frequencies $v=375 \mathrm{GHz}, 600 \mathrm{GHz}, 700 \mathrm{GHz}$, and $857 \mathrm{GHz}$ are relevant to study the importance of SZ effect relativistic corrections.

Using the X-ray observations of the Bullet cluster and Abell 2219 (Million \& Allen 2009), which are massive merging galaxy clusters, we calculated the SZ intensity maps at low and high frequencies and have checked that the morphologies of the SZ intensity maps at low and high frequencies are different. We will address this problem more specifically in a forthcoming paper.

\section{Observational considerations}

We briefly discuss here the detectability of the electron DF through SZ effect observations in the optimal frequency sample studied above.
We find that the difference of the SZ effect signals predicted for the Juttner and the Maxwell-Boltzmann DFs at 375, 600, 700 and $857 \mathrm{GHz}$ can be only marginally appreciated by the PLANCK-HFI instrument with the nominal 2-year survey sensitivity (at $1 \sigma$ level). Indeed, while the DF difference in the predicted SZ effect intensities can be determined at 375 and $600 \mathrm{GHz}$ channels at the $\sim 3.5 \sigma$ and $\sim 3.4 \sigma$ level, it is impossible to determine it for the highest channels, i.e. 700 and $857 \mathrm{GHz}$ of PLANCK-HFI. Note also that the Bullet cluster is almost unresolved for the PLANCK-HFI frequency channels.

However, the 600 and $857 \mathrm{GHz}$ frequency channels are also covered by the HERSCHEL-SPIRE instrument, whose sensitivity is sufficient to detect the predicted SZ effect intensity difference necessary to distinguish between the Juttner and MaxwellBoltzmann DFs at 600 and $857 \mathrm{GHz}$. We also notice that the Bullet cluster is a fully resolved source for the HERSCHELSPIRE instrument.

An important point to be addressed is the impact of the confusion noises at these high-frequency channels to distinguish the DF effects in the SZ effect observations. Relevant sources of confusion for SZ effect observations at high frequencies are the CMB fluctuation, the unresolved point-like millimeter sources emission, and the diffuse emission from the Galaxy. For an experiment that can spatially resolve the Bullet cluster, the $\mathrm{CMB}$ confusion is negligible on sub-arcmin scales, while the unresolved point-like source emission and the Galaxy emission increase with frequency, which provides the major confusion sources. For cluster temperatures on the order of $15 \mathrm{keV}$ (as in the Bullet cluster), the diffuse Galaxy emission is the dominant confusion noise.

Therefore, a multi-frequency observational strategy is required to properly monitor and subtract the confusion noises that have a different frequency behavior with respect to the SZ effect spectrum. Nonetheless, the confusion noise level adds up to the instrument noise to reduce the possibility of the SZ effect observations in order to derive the electron DF. Longer exposure with spectroscopic instruments operating in this high-frequency range are required to reach a precision level on the order of $\sim 0.1 \%$ to achieve a good statistical control of the systematics and a good statistical confidence level of the parameters necessary to reconstruct the electron DF.

\section{Conclusions}

The SZ effect is an important tool for cosmology and for the astrophysical study of clusters of galaxies (for a review, see Birkinshaw 1999). It measures the pressure of an electron population integrated along the line of sight as long as free electrons are non-relativistic. Relativistic corrections of the SZ effect allow us to measure the plasma temperature.

Although it has already been noticed that the use of the correct relativistic equilibrium distribution is essential for the proper interpretation of measurements of the SZ effect, no studies of this problem have been performed in detail so far.

The relativistic kinetic theory, on which the DF derivation is based, is still a subject of numerous debates. The relativistic analogue of the Maxwell-Boltzman velocity DF has been proposed by Juttner (1911). However, alternatives to a Juttner DF have been discussed by Horwitz et al. (1989) and, recently, by Lehmann (2006) and Dunkel \& Hanggi (2007).

In this paper, we showed how to separate the CMB distortions (caused by the SZ effect) that are caused by a departure from the diffusive approximation given by Kompaneets (1957) 
from those that are caused by using a relativistic correct DF instead of a Maxwell-Boltzman DF. We propose here a method based on Fourier analysis to derive a velocity DF of electrons by using SZ observations at four frequencies.

We found that the SZ intensity change owing to using a relativistic correct DF instead of a Maxwell-Boltzman DF contributes a significant part to the total relativistic corrections of the SZ effect and is more significant at lower temperatures $\approx 5 \mathrm{keV}$ than that at higher temperatures $\approx 15 \mathrm{keV}$. We conclude that the value of the SZ intensity change owing to using a relativistic correct DF instead of a Maxwell-Boltzman DF will be much higher in hot galaxy clusters because the value of the relativistic SZ corrections is proportional to $T_{\mathrm{e}}^{5 / 2}$.

We proposed a method to derive the DF of electrons using SZ multi-frequency observations of massive galaxy clusters with high plasma temperatures. Using a Fourier analysis we found that the approximate electron DF represented by six Fourier cosine terms accurately describes the relativistic Juttner DF. By means of SZ intensity measurements at four frequencies we showed how to derive the approximate DF of electrons. To find a suitable sample of four frequencies for deriving the DF of electrons, we studied different samples of frequencies. We found that the best sample includes high frequencies $v=375$, $600,700,857 \mathrm{GHz}$, while the worst sample is in the frequency range $300-400 \mathrm{GHz}$ because the matrix $M_{\lambda k}$, which is determined in Sect. 3, is ill-conditioned for this frequency range. In the case of the frequency sample of $v=375,600,700,857 \mathrm{GHz}$ to derive a DF of electrons the allowable uncertainties in the SZ intensities are about three orders of magnitude larger than those found for the frequency sample $v=300,320,340,360 \mathrm{GHz}$. Using Monte-Carlo simulations of SZ observations with the SZ intensity observational uncertainty of $0.1 \%$, we showed that it is possible to distinguish Juttner and Maxwell-Bolzman DFs by means of these SZ observations. Therefore, the SZ effect provides us with a promising method to study DFs of electrons in massive galaxy clusters that contain hot plasmas with temperatures $\simeq 15 \mathrm{keV}$.

We considered in our analysis the relaxation of a system of electrons with Coulomb interactions and conclude that the Juttner distribution function is an appropriate approximation to the universal (equilibrium) electron distribution in hot merging clusters.

We applied this method to derive the DF of electrons using multi-frequency SZ observations in galaxy clusters where hard $\mathrm{X}$-ray tails were detected. We demonstrated the ability of SZ multi-frequency observations to derive the electron DF for the Bullet cluster and found that a precision of SZ intensity measurements of $\approx 0.1 \%$ is required. This method is independent of those proposed by Blasi et al. (2000), Shimon \& Rephaeli (2002), and Colafrancesco et al. (2009), and can be used to distinguish among different interpretations of the X-ray excess. Although our method requires a higher precision of SZ observations compared with other methods, it permits us to directly study electron DFs rather than studying the supplementary electron pressure caused by the presence of quasi-thermal particles. This is an important advantage of our method.

Using the 3D hydrodynamic numerical simulations of a hot merging galaxy cluster, we also demonstrated that the SZ spatial intensity maps of the simulated hot merging galaxy cluster at frequencies of $375 \mathrm{GHz}, 600 \mathrm{GHz}, 700 \mathrm{GHz}$, and $857 \mathrm{GHz}$ are different. This is because of the important role of SZ relativistic corrections at high frequencies. New SZ multi-frequency measurements with a high spatial resolution should confirm our conclusion.

The next generation of SZ effect experimental techniques (as those outlined in Colafrancesco \& Marchegiani 2010) are needed to reach the required high sensitivity with the purpose of studying electron DFs by means of multi-frequency SZ observations.

Acknowledgements. We are grateful to Joseph Silk and Vladimir Dogiel for valuable suggestions and discussions and thank the referee for valuable suggestions.

\section{References}

Ajello, M., Rebusco, P., Cappelluti, N., et al. 2010, ApJ, 725, 1688

Akahori, T., \& Yoshikawa, K. 2010, PASJ, 62, 335

Birkinshaw, M. 1999, Phys. Rep., 310, 97

Blasi, P. 2000, ApJ, 532, L9

Blasi, P., Olinto, A. V., \& Stebbins, A. 2000, ApJ, 535, L71

Bryan, G. L., \& Norman, M. L. 1998, ApJ, 495, 80

Challinor, A., \& Lasenby, A. 1998, ApJ, 499, 1

Clarke, T. E., Kronberg, P. P., \& Böhringer, H. 2001, ApJ, 547, L111

Colafrancesco, S., \& Marchegiani, P. 2010, A\&A, 520, A31

Colafrancesco, S., Marchegiani, P., \& Palladino, E. 2003, A\&A, 397, 27

Colafrancesco, S., Prokhorov, D., \& Dogiel, V. 2009, A\&A, 494, 1

Cubero, D., Casado-Pascual, J., Dunkel, J., Talkner, P., \& Hänggi, P. 2007, Phys. Rev. Lett., 99, 170601

Dogiel, V. A., Colafrancesco, S., Ko, C. M., et al. 2007, A\&A, 461, 433

Dunkel, J., \& Hanggi, P. 2007, Physica A, 374, 559

Fusco-Femiano, R., Dal Fiume, D., Feretti, L., et al. 1999, ApJ, 513, L21

Fusco-Femiano, R., Orlandini, M., Brunetti, G., et al. 2004, ApJ, 602, L73

Hansen, S. H., Pastor, S., \& Semikoz, D. V. 2002, ApJ, 573, L69

Horwitz, L. P., Shashoua, S., \& Schieve, W. C. 1989, Physica A, 161, 300

Itoh, N., Kohyama, Y., \& Nozawa, S. 1998, ApJ, 502, 7

Juttner, F. 1911, Ann. Phys. (Leipzig), 34, 856

Kaastra, J. S., Bykov, A. M., \& Werner, N. 2009, A\&A, 503, 373

Kompaneets, A. S. 1957, Soviet Phys. JETP, 4, 730

Landau, L. D., \& Lifshitz, E. M. 1976, Statistical Physics, Courses of Theoretical Physics (Nauka), 5

Lehmann, E. 2006, J. Math. Phys., 47, 023303

Liang, H., Hunstead, R. W., Birkinshaw, M., \& Andreani, P. 2000, ApJ, 544, 686

Liang, H., Dogiel, V., \& Birkinshaw, M. 2002, MNRAS, 337, 567

MacDonald, W. M., \& Rosenbluth, M. N. 1957, Phys. Rev., 107, 350

Markevitch, M., Gonzalez, A. H., David, L., et al. 2002, ApJ, 567, L27

Million, E. T., \& Allen, S. 2009, MNRAS, 399, 1307

Montakhab, A., Ghodrat, M., \& Barati, M. 2009, Phys. Rev. E., 79, 031124

Peano, F., Marti, M., Silva, L. O., \& Coppa, G. 2009, Phys. Rev. E, 79, 025701

Petrosian, V. 2001, ApJ, 557, 560

Petrosian, V., \& East, W. 2008, ApJ, 682, 175

Petrosian, V., Madejski, G., \& Luli, K. 2006, ApJ, 652, 948

Pointecouteau, E., Giard, M., \& Barret, D. 1998, A\&A, 336, 44

Prokhorov, D. 2009, A\&A 508, 6974

Prokhorov, D. A., \& Durret, F. 2007, A\&A, 474, 375

Prokhorov, D. A., Durret, F., Dogiel, V. A., et al. 2009, A\&A, 496, 25

Prokhorov, D., Dubois, Y., \& Nagataki, S. 2010a, A\&A, 524, A89

Prokhorov, D., Antonuccio-Delogu, V., \& Silk, J. 2010b, A\&A, 520, A106

Rephaeli, Y. 1995, ApJ, 445, 33

Rephaeli, Y., Nevalainen, J., Ohashi, T., \& Bykov, A. M. 2008, SSRv, 134, 71

Rossetti, S., \& Molendi, S. 2004, A\&A, 414, 41

Sarazin, C. L. 1986, Rev. Mod. Phys., 58, 1

Sarazin, C. L. 2002, Merging Processes in Clusters of Galaxies, ASSL, 272, 1 (Kluwer Academic Publisher)

Sarazin, C. L., \& Kempner, J. C. 2000, ApJ, 533, 73

Shimon, M., \& Rephaeli, Y. 2002, ApJ, 575, 12

Sunyaev, R. A., \& Zel'dovich, Ya. B. 1980, ARA\&A, 18, 537

Wolfe, B., \& Melia, F. 2008, ApJ, 675, 156

Wright, E. L. 1979, ApJ, 232, 348

Zemcov, M., Rex, M., Rawle, T. D., et al. 2010, A\&A, 518, L16 